\title{
Granatapfel - lecker, aber doch ineffektiv?
}

Gleich zwei aktuelle Metaanalysen befassen sich mit der gesundheitlichen Wirkung von Zubereitungen aus Granatäpfeln - und sie kommen zu einem in dieser Hinsicht eher bescheidenen Urteil.

Wegen ihres hohen Gehalts an einer Vielzahl von Inhaltsstoffen stehen die Früchte des Granatapfelbaums Punica granatum L. seit einiger Zeit im Interesse all jener Forschungsgruppen, die auf der Suche nach natürlichen Antioxidanzien und pharmakologisch aktiven Polyphenolen sind. Zahlreiche Studien in vitro und in vivo scheinen den seit vielen Jahrhunderten in der fernöstlichen und mediterranen Heilkunde praktizierten Einsatz von Granatäpfeln, v.a. deren Saft und Samen, zu bestätigen. Die gesamte Pflanze von der Wurzel über die Rinde, die Blätter und die Früchte enthält potenziell aktive Inhaltsstoffe der Substanzklassen der Flavonoide, kondensierte und lösliche Tannine sowie andere polyphenolische Komponenten. Für sie wurden isoliert oder im Zusammenspiel als Gesamtextrakt positive kardiovaskuläre, entzündungshemmende, antioxidative und chemopräventive Effekte nachgewiesen. Dabei übertrifft die Vielfalt an Polyphenolen im Granatapfel in ihrer antioxidativen Wirkung deutlich diejenige von grünem Tee oder Rotwein. Untersuchungen an Zellkulturen und Tiermodellen belegten positive Effekte von Punica-Extrakten bei Erkrankungen wie Diabetes mellitus, Arteriosklerose, Krebserkrankungen von Darm, Brust oder Prostata, AlzheimerDemenz oder Aids.

Der großen Anzahl an In-vitro- und In-vivo-Studien steht jedoch eine relativ überschaubare Anzahl mehr oder weni-

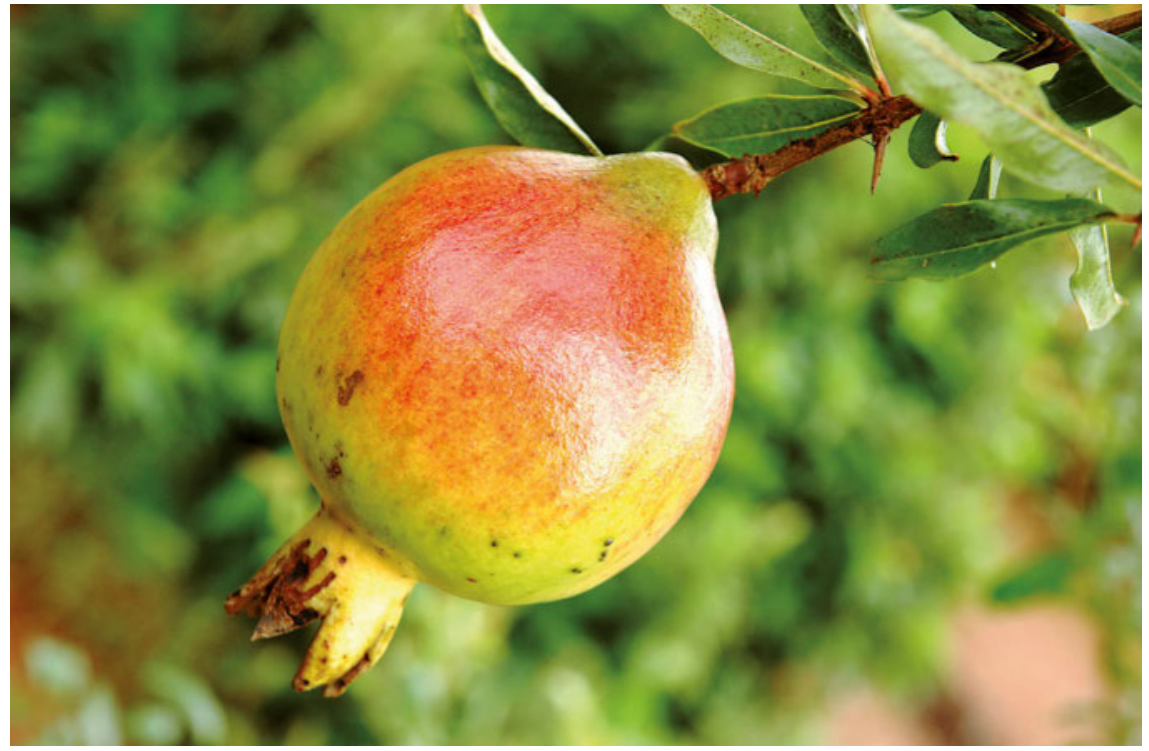

Randvoll mit Antioxidanzien, aber anscheinend ohne diesbezügliche Wirkung. @ S. Shankar

ger gut durchgeführter placebokontrollierter klinischer Studien mit einem randomisierten, doppelt oder einfach verblindeten Design gegenüber. Diese wurden nun in zwei Metaanalysen ausgewertet: Eine Analyse befasste sich mit der in den gepoolten klinischen Studien untersuchten Auswirkung von PunicaZubereitungen auf das C-reaktive Protein (CRP) als Marker für Entzündungsvorgänge im Körper. Die andere Untersuchung verglich die Wirkung von Granatäpfeln auf das Lipidprofil der Studienteilnehmer und damit indirekt auf die kardiovaskulären Risiken.

\section{CRP-Werte zeigen sich unbeeindruckt}

Nur 5 Studien erfüllten die vorab festgelegten Einschlusskriterien für die Metaanalyse zum Einfluss von Granatapfel auf den CRP-Plasmaspiegel. Sie lieferten die Daten von insgesamt 427 Patienten; 216 hatten über einen Zeitraum von 2 Wochen bis 18 Monaten - je nach Studie Punica-Saft oral zu sich genommen und 211 waren Kontrollpersonen. Dabei zeigte die statistische Auswertung, u.a. auch mittels Metaregressionsberechnung, dass der Pflanzensaft keinen signifikanten Effekt bezüglich der Reduktion der CRPWerte im Vergleich zu Placebo hervorruft und damit das in vitro und in vivo ermittelte antioxidative Potenzial sich klinisch diesbezüglich nicht auswirkt - und zwar unabhängig von Dauer und Dosis des Saftkonsums. Nach Ausschluss der einzigen Studie mit US-amerikanischer Bevölkerung ergab sich für die verbliebenen 4 Studien aus dem Iran ein Rückgang der Plasma-CRP-Werte, wenn auch nicht signifikant. Dies ließe sich eventuell mit genetischen Varianten des CRP-Gens erklären. 


\section{Wie beeinflusst Granatapfel- saft das Lipidprofil?}

Anhand ähnlich strenger Einschlusskriterien wie in der Metaanalyse zum CRP konnten insgesamt 12 randomisiert-kontrollierte klinische Studien ausgewertet werden, die sich mit dem Einfluss von Punica-Saft auf die Lipidparameter über einen Zeitraum von 10 Tagen bis zu einem Jahr befassten - allerdings hatten nicht alle Studien auch hyperlipidämische Patienten eingeschlossen. Wie beim CRP ergab auch hier die Metaregression keinen bedeutenden Effekt auf die Konzentration der Plasmalipide im Vergleich zu Placebo hinsichtlich der konsumierten Saftmenge. In Bezug auf die Dauer der Einnahme zeigte sich kein statistisch signifikanter Unterschied zwischen Placebo und Granatapfelsaft bei den Werten für Gesamt-, LDL- und HDL-Cholesterin, jedoch eine inverse Assoziation mit den Veränderungen der Triglyzeridwerte.

\section{Mögliche Erklärungen}

Zum einen waren in den in die Metaanalysen eingeschlossenen Studien die Zu- sammensetzungen der Granatapfelsäfte nicht einheitlich, sowohl hinsichtlich der Herkunft und Anbaubedingungen als auch in Bezug auf die Weiterverarbeitung. Dies kann in einer deutlich verschiedenen Zusammensetzung der Inhaltsstoffe resultieren und damit in einer veränderten Bioverfügbarkeit und/oder unterschiedlichen pharmakologischen Auswirkungen. Auch die Dosierungen waren uneinheitlich und wegen fehlender Dosisfindungsstudien evtl. auch unterdosiert. Zudem könnte die Studiendauer bei allen klinischen Studien noch zu kurz gewesen sein, um die Plasmawerte so deutlich $\mathrm{zu}$ beeinflussen, wie es durch die Vorläuferstudien in vitro und in vivo zu erwarten gewesen wäre.

\section{Fazit}

Die gemeinsame Auswertung von randomisierten placebokontrollierten Studien über die gesundheitlichen Effekte von Granatapfel-Zubereitungen kann die positiven antioxidativen und antiatherosklerotischen Ergebnisse von Untersuchungen in vitro und in vivo nicht im klinischen Setting bei Menschen bestäti- gen. Weder zeigten sich statistisch relevante Wirkungen auf den Entzündungsmarker C-reaktives Protein noch auf das Plasma-Lipidprofil. Hier sind also weitere Studien mit detaillierterem Design bezüglich Bioverfügbarkeit oder optimaler Dosierung bzw. Einnahmedauer erforderlich.

Ulrike Andres, Ginsheim

Literatur

1 Sahebkar A, Gurban C, Serban A et al. Effects of supplementation with pomegranate juice on plasma C-reactive protein concentrations: A systematic review and meta-analysis of randomized controlled trials. Phytomedicine 2016; 23: 1095-1102

2 Sahebkar A, Simental-Mendía LE, Giorgini P et al. Lipid profile changes after pomegranate consumption: A systematic review and metaanalysis of randomized controlled trials. Phytomedicine 2016; 23: 1103-1112 